\title{
ON THE PROBLEM OF CLASSIFYING INFINITE CHAINS IN PROJECTIVE AND ORTHOGONAL GEOMETRY
}

\author{
HERBERT GROSS and HANS A. KELLER*)
}

\section{Introduction}

In [8] a classification of subforms of diagonal forms (symmetric bilinear over an algebraically closed field or else alternate) in dimensions less than $\boldsymbol{\aleph}_{\omega_{0}}$ was obtained. Trying to push the classification up to dimension $\aleph_{\omega_{0}}$, and beyond, one discovers that the recursive methods developed for dimensions below $\boldsymbol{\aleph}_{\omega_{0}}$ get stuck.

The reason is the following. The classification (up to isometry) of subforms of diagonal forms $\Phi$ is equivalent to the classification of certain descending chains of subspaces in the vector space $E$ that carries $\Phi$ (the second classification is modulo the action of the orthogonal group of the quadratic space $(E, \Phi))$. Now, if $\operatorname{dim} E \geqq \aleph_{\omega_{0}}$ then these chains are infinite and, with infinite descending chains, a variety of new difficulties makes its appearance. Some of these difficulties are of a metric nature, others are of a projective kind, and still others are of an entirely topological character. We shall show that these aspects are largely independent one from the other.

A somewhat similar situation is encountered in the classification theory of abelian groups (Ulm's Theorem) in the uncountable case. However, the analogy does not carry very far. The group structure is trivial in the vector space case; on the other hand there are various additional structures originating with the form.

The classification of subforms of diagonal forms is a special case of a mapping problem which has been the source of many new results during recent years (refer to $[5,8,10,15,17])$. We begin our paper by formulating a projective version of this mapping problem and by giving a solution (Theorem 1) of it when no infinite descending chains interfere.

AMS (MOS) subject classification (1970). Primary 10 C 01, 15 A 63, 51 D 25, 51 F 20.

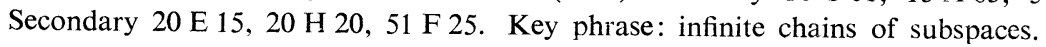

*) This work was done while the second author was a Fellow of the Schweizerische Nationalfonds (Grant Nr. 2. 627-0.80). 
Next we construct a series of examples which illustrate the various kinds of difficulties met in the classification of infinite descending chains $\mathscr{C}$ under the action of $\mathrm{GL}(E)$ and certain of its subgroups. The examples are such that the orbits of $\mathscr{C}$ split into proper suborbits as we pass from one classification to the next.

We then treat cases where the mapping problem in the metric setting can be solved even in the presence of infinite descending chains (Theorems 6 and 7). We apply the results to the classification of subforms of diagonal forms ("prediagonal forms"). We mention in particular Theorem 11 which gives a topological characterization of the isometry types of prediagonal spaces of small uncountable dimensions.

In order to make our constructions as perspicuous as possible we stick to the simplest cases suitable for our purposes. This means restriction to the consideration of $\omega_{0}$-chains. We are aware of the fact that more gadgets can be thought up for longer chains; however, a firm grasp of the $\omega_{0}$-chains is pre-eminent. We shall consider chains of the sort

$$
\mathscr{C}=\left(V_{i}\right)_{i<\omega_{0}}, \quad V_{i} \supseteq V_{i+1}, \quad \operatorname{dim} V_{i} / V_{i+1}=1, \quad i<\omega_{0}
$$

where the $V_{i}$ are linear subspaces in a vector space $E$ of dimension $\aleph_{1}\left(2 \aleph_{0}\right.$ whenever more convenient) in which case we shall assume that $\bigcap_{i} V_{i}=(0)$ ("reduced chain"), or else in a space $E$ of dimension $\aleph_{\omega_{0}}$ in which case we set $V_{\infty}:=\bigcap_{i} V_{i}$. Furthermore, we shall use the following notations:

$E \quad$ vector space over a commutative field of arbitrary characteristic,

$\mathscr{L}(E) \quad$ lattice of all linear subspaces of $E$,

$\mathscr{V}$ sublattice of $\mathscr{L}(E)$,

$\mathscr{C}$ descending chain in $\mathscr{L}(E)$,

DCC descending chain condition,

$\mathscr{G} \quad$ filter in $\mathscr{L}(E)$,

$\varrho(\mathscr{G}), \varrho(\mathscr{C})$ the linear topology on $E$ with zero-neighbourhood filter basis $\mathscr{G}, \mathscr{C}$, respectively,

$\varrho X \quad$ closure of $X \subset E$ with respect to the topology $\varrho$,

$(E, \Phi) \quad \Phi$ symmetric or antisymmetric bilinear form on $E$,

$d \cdot E$ external orthogonal sum of $d$ copies of $(E, \Phi), d$ any cardinal,

$\tau_{\gamma}(\Phi), \sigma_{\gamma}(\Phi)$ certain linear topologies on $E$, related by $\sigma_{;+1}(\Phi)=\tau_{;}(\Phi), \gamma$ any ordinal,

$\tau_{\gamma} X, \sigma_{\gamma} X \quad$ closure of $X \subset E$ with respect to $\tau_{\gamma}(\Phi), \sigma_{\gamma}(\Phi)$, respectively,

$\widetilde{E}^{\tilde{E}}$ completion of the uniform space $\left(E, \tau_{0}(\Phi)\right)=\left(E, \sigma_{1}(\Phi)\right)$.

Refer to [9] for terminology and basic facts on infinite dimensional sesquilinear forms. 


\section{A mapping problem of projective geometry}

I. 1. The Problem (henceforth referred to as "the mapping problem"). Let $E$ be an infinite dimensional vector space and

$$
\eta: \mathscr{V} \rightarrow \mathscr{V}^{\prime}
$$

a lattice isomorphism between sublattices of $\mathscr{L}(E)$. When is $\eta$ induced by an element of $\mathrm{GL}(E)$ (the group of linear automorphisms of $E$ )? An obvious requirement is that $\eta$ preserve rank, i.e. all "indices" $\operatorname{dim} Y \mid X$ for $X \subset Y$ in $\mathscr{V}$; cf. Remark 8. It is furthermore no restriction to assume $\mathscr{V}, \mathscr{V}^{\prime}$ at the outset to be complete as sublattices of $\mathscr{L}(E)$. Quite often we shall assume $\mathscr{V}^{\prime}$ to be a subset in $\mathscr{L}\left(E^{\prime}\right), E^{\prime}$ a vector space isomorphic to $E$ which makes for more systematic notations.

I. 2. The lattices admitted. We shall study the mapping problem for various classes of lattices. In order to concentrate on the difficulties with descending chains we shall always restrict ourselves to the consideration of distributive lattices $\mathscr{V}$; they will be subjected to one more condition below (namely (4)). We assume furthermore that $(0) \in \mathscr{V}$ and $E \in \mathscr{V}$.

I. 3. The recursive setup for the solution. It is natural to visualize the required mapping $T: E \rightarrow E^{\prime}$ as the union of partial mappings. We therefore introduce the family $\mathscr{F}_{0}=\mathscr{F}_{0}(\eta)$ of all linear isomorphisms $\varphi: X \rightarrow X^{\prime}, X \in \mathscr{L}(E), X^{\prime} \in \mathscr{L}\left(E^{\prime}\right)$, which satisfy the condition

$$
\varphi(X \cap A)=X^{\prime} \cap \eta A \text { for all } A \in \mathscr{V} .
$$

One then looks for chains $\left(\varphi_{\gamma}: X_{\gamma} \rightarrow X_{\gamma}^{\prime}\right)_{\gamma \in \Gamma}$ in $\mathscr{F}_{0}$ with

$$
\bigcup_{\Gamma} X_{\gamma}=E, \quad \bigcup_{\Gamma} X_{\gamma}^{\prime}=E^{\prime}
$$

The limit map of such a chain solves the mapping problem.

1. 4. Solution of the problem for lattices with the descending chain condition (DCC). In the case of certain lattices it is possible to restrict oneself to the subfamily $\mathscr{F}_{1}(\eta)$ of all $\left(\varphi: X \rightarrow X^{\prime}\right) \in \mathscr{F}_{0}(\eta)$ with the two properties:

$$
\begin{array}{ll}
(X+A) \cap(X+B)=X+(A \cap B) ; & A, B \in \mathscr{V} \\
\left(X^{\prime}+A^{\prime}\right) \cap\left(X^{\prime}+B^{\prime}\right)=X^{\prime}+\left(A^{\prime} \cap B^{\prime}\right): & A^{\prime}, B^{\prime} \in \mathscr{V}^{\prime}
\end{array}
$$

This is shown by

Lem ma 1. Let $\mathscr{V}$ be a complete and distributive sublattice of $\mathscr{L}(E)\left(\operatorname{dim} E=\omega_{\alpha}\right)$ which satisfies DCC. Then there exists a set of generators $\left(x_{v}\right)_{v<\omega_{\alpha}}$ of $E$ with the following property: If $X_{x}:=\operatorname{span}\left\{x_{v} \mid v<x\right\}$ then for all $x<\omega_{\alpha}$ and all $A, B \in \mathscr{V}$ we have $\left(X_{\varkappa}+A\right) \cap\left(X_{\varkappa}+B\right)=X_{\varkappa}+(A \cap B)$. 
Thus, if there is a solution to the mapping problem at all then it can be obtained as a limit map of a chain in $\mathscr{F}_{1}(\eta)$.

Proof of Lemma 1. Let $\left(e_{\lambda}\right)_{\lambda<\omega_{\alpha}}$ be a basis of $E$. Assume that for $v<\varkappa$ $x_{v}$ is defined such that (3) holds for all $X_{\mu}, \mu \leqq x$. Let $\lambda$ be the smallest index with $e_{\lambda} \notin X_{\varkappa}$. The set

$$
\mathscr{M}=\mathscr{M}\left(e_{\lambda}, X_{\varkappa}\right):=\left\{A \in \mathscr{V} \mid e_{\lambda} \in X_{\varkappa}+A\right\}
$$

is a filter by the induction assumption, hence a principal filter by DCC, say $\mathscr{M}=(D)$. First case: $D$ is + -irreducible so $\mathscr{M}$ is a prime filter). We decompose $e_{\lambda}$, $e_{\lambda}=d+x$ where $d \in D$ and $x \in X_{\chi}$. Set $x_{\chi}:=d$; one verifies just as in [9, p. 118] that (3) holds again for $X=X_{x+1}$. Second case: $D$ is + -reducible. It is here that we need a consequence of DCC, namely

$$
\text { compact elements are joins of join-irreducibles*) }
$$

The case is now reduced to the former as in [9,p.119]: one finds $x_{x}, x_{x+1}, \ldots, x_{x+m}$ such that the finitely many $X_{x+1}, \ldots, X_{x+m+1}$ satisfy (3) and such that $e_{\lambda} \in X_{x+m+1}$.

Conversely, we now show that a solution of the mapping problem always exists under the provisos stated:

Theorem 1. Let $\mathscr{V}, \mathscr{V}^{\prime}$ be complete, distributive sublattices of $\mathscr{L}(E)$ and $\mathscr{L}\left(E^{\prime}\right)$ satisfying DCC and (4); here $E, E^{\prime}$ are vector spaces of arbitrary (equal) dimension over the same field. Each rank preserving lattice isomorphism $\eta: \mathscr{V}^{\prime} \mathscr{V}^{\prime}$ comes from a projectivity, i.e. is induced by a linear bijection $T: E \rightarrow E^{\prime}$.

Proof. The union of any ascending chain of elements in $\widetilde{F}_{1}(\eta)$ belongs to $\mathscr{F}_{1}(\eta)$ again ("chain-property"). Furthermore, $\mathscr{F}_{1}(\eta)$ enjoys the "Ping Pongproperty" (PP): for each $\left(\varphi: X \rightarrow X^{\prime}\right) \in \mathscr{F}_{1}(\eta)$ and each $x \in E\left(x^{\prime} \in E^{\prime}\right)$ there exist $\left(\varphi_{1}: X_{1} \rightarrow X_{1}^{\prime}\right) \in \mathscr{F}_{1}(\eta)$ with $x \in X_{1}\left(x^{\prime} \in X_{1}^{\prime}\right)$ and $\varphi_{1} \supseteqq \varphi$. Starting with the zero map $\left(\varphi_{0}:(0) \rightarrow(0)\right) \in \mathscr{F}_{1}(\eta)$ we may therefore, by transfinite recursion, obtain a chain $\left(\varphi_{\gamma}: X_{\gamma} \rightarrow X_{\gamma}^{\prime}\right)_{\gamma \in \Gamma}$ in $\mathscr{F}_{1}(\eta)$ that satisfies (2) just as in the denumerable case $[9$, Chapter IV].

Remark 1. The core of the preceding proof is the (PP) property of $\mathscr{F}_{1}(\eta)$ : one has to extend a partial mapping $\varphi: X \rightarrow X^{\prime}$ such that the extension satisfies (1). According to [9, Chapter IV] this is always possible if the filter

$$
\mathscr{M}(x, X):=\{A \in \mathscr{V} \mid x \in X+A\}
$$

happens to be a principal filter. If, in the following, we also succeed with the recursive setup (I.3) in some cases where the lattices do not satisfy DCC then, invariably, it is because we have discovered a way to monitor the course of recursion

*) For complete sublattices of $\mathscr{L}(E)$ distributivity together with (4) is equivalent to complete distributivity. A proof is outlined in [9, Chapter IV, Section 2]. 
in such a manner that, at each step, the arising filters $\mathscr{M}$ turn out to be principal filters.

I. 5. Infinite descending chains lead to new invariants. Let $\mathscr{C} \subseteq \mathscr{L}(E)$ be a descending $\omega_{0}$-chain and $\varrho(\mathscr{C})$ the linear topology on $E$ with $\mathscr{C}$ as a zero neighbourhood basis. Assume that the lattice $\mathscr{V}$ in our mapping problem contains such a $\mathscr{C}$; then by (1) each element in $\mathscr{F}_{0}(\eta)$ is a homeomorphism with respect to $\varrho(\mathscr{C})$ and $\varrho(\eta \mathscr{C})$; likewise, each linear isomorphism $T: E \rightarrow E^{\prime}$ which induces $\eta$ is a homeomorphism $(E, \varrho(\mathscr{C})) \cong\left(E^{\prime}, \varrho(\eta \mathscr{C})\right)$. Hence the importance of the topologies $\varrho(\mathscr{C})$ (for all $\mathscr{C} \subset \mathscr{V}$ ) for our mapping problem.

We first convince ourselves that there are enough such chains in $\mathscr{L}(E)$ for our discussions.

Lemma 2. Let the $k$-space $E$ have dimension $a:=|k|^{\aleph_{0}}$ and let $0 \neq r<\omega_{0}$ be fixed. In $\mathscr{L}(E)$ there are precisely $\mid k^{\mid a}$ chains $\mathscr{C}$ with property (0) and such that (i) the spaces $(E, \varrho(\mathscr{C}))$ are mutually non-homeomorphic, (ii) if $\bar{E}$ is the compleiion of the uniform space $(E, \varrho(\mathscr{C}))$ then $\operatorname{dim} \bar{E} / E=r$ for all these $\mathscr{C}$.

Proof. We begin by estimating the number of $\mathscr{C}^{\prime}$ such that $\left(E, \varrho\left(\mathscr{C}^{\prime}\right)\right)$ is homeomorphic to a fixed $(E, \varrho(\mathscr{C}))$. Let $\left(h_{i}\right)_{i \geqq 1}$ be a basis of a $\boldsymbol{\kappa}_{0}$-dimensional $k$-space $H$. In the algebraic dual $H^{*}$ pick a subspace $E$ such that the chain $\mathscr{C}_{E}=\mathscr{C}:=\left(V_{i}\right)_{i \in \omega_{0}}, \quad V_{i}:=\left\{e \in E \mid\left\langle e, h_{1}\right\rangle=\ldots=\left\langle e, h_{i}\right\rangle=0\right\}$ satisfies (0). Let $E^{\prime} \subset H^{*}$ and $\mathscr{C}^{\prime}=\mathscr{C}_{E}^{\prime}$ be analogous objects. The weak linear topology $\sigma(E, H)$ of the pairing $\langle E, H\rangle$ is precisely $\varrho(\mathscr{C})$. One now proves with the usual arguments that cach linear homeomorphism

$$
\begin{aligned}
& f:(E, \varrho(\mathscr{C})) \rightarrow\left(E^{\prime}, \varrho\left(\mathscr{C}^{\prime}\right)\right) \text { has the form } f=\left.g^{*}\right|_{E}, \\
& \text { where } g^{*} \in G L\left(H^{*}\right) \text { is the adjoint of a } g \in G L(H) .
\end{aligned}
$$

The adjoint $g^{* *}: H^{* *} \rightarrow H^{* *}$ then maps the $r$-dimensional orthogonals $E^{\perp} \subset H^{* *}$, $E^{\prime \perp} \subset H^{* *}$ onto each other. Hence the number of $\left(E, \varrho\left(\mathscr{C}^{\prime}\right)\right)$, homeomorphic to a fixed $(E, \varrho(\mathscr{C}))$, is less or equal to $|\mathrm{GL}(H)|=a$. On the other hand there are precisely $\mid k^{a} \geqq 2^{a}>a$ subspaces $Y \subset H^{* *}$ of fixed dimension $r$. If we think of $H^{*}$ as a subspace of $H^{* *}$, then we can also pick $\mid k^{a}$ such $Y$ which, in addition, satisfy $Y \cap H=(0)$. These $Y$ appear as orthogonals $E^{\perp}$ of suitable subspaces $E \subset H$, i.e. $E$ such that the chains $\mathscr{C}_{E}$ which they define all satisfy (0). Hence the assertion of the lemma.

I. 6. A linear homeomorphism $(E, \varrho(\mathscr{C})) \cong\left(E, \varrho\left(\mathscr{C}^{\prime}\right)\right)$ does not imply that $\mathscr{C}$ and $\mathscr{C}^{\prime}$ are in the same orbit under GL $(E)$. In other words, the projective classification of the chains $\mathscr{C} \subset \mathscr{L}(E)$ is finer than the classification via their associated topologies $\varrho(\mathscr{C})$. We shall prove

Theorem 2. Let $E$ and $\bar{E}$ be as in Lemma 2 and $\operatorname{dim} \bar{E} / E=1$. We can spacify $\mathscr{C}, \mathscr{C}^{\prime}$ with property $(0)$ such that $(E, \varrho(\mathscr{C}))$ and $\left(E, \varrho\left(\mathscr{C}^{\prime}\right)\right)$ are linearly homeomorphic but no $T \in \mathrm{GL}(E)$ can map $\mathscr{C}$ onto $\mathscr{C}^{\prime}$. 
Proof. Let $\left(h_{2 i}\right)_{i \geqq 1},\left(h_{2 i-1}\right)_{i \geqq 1}$ be bases of two $\aleph_{0}$-dimensional $k$-spaces $H_{+}, H_{-}$. We set $H:=H_{-} \oplus H_{+}$and choose in its algebraic dual $H^{*} \cong H_{-}^{*} \oplus H_{+}^{*}$ a hyperplane $E:=H_{-}^{*} \oplus L, L \subset H_{+}^{*}$ in such a way that the chain $\mathscr{C}=\left(V_{i}\right)_{i \geqq 0}$ with $V_{i}:=\left\{e \in E \mid\left\langle e, h_{1}\right\rangle=\ldots=\left\langle e, h_{i}\right\rangle=0\right\}$ satisfies (0). Let $g \in \mathrm{GL}(H)$ be the operation which interchanges $h_{2 i}$ and $h_{2 i-1}$. We set $E^{\prime}:=g^{*}(E)$,

$$
E^{\prime}=M \oplus H_{+}^{*}, \quad M=g^{*}(L) \subset H_{-}^{*} .
$$

Obviously, the chain $\mathscr{C}^{\prime}:=\left(V_{i}^{\prime}\right)_{i \geqq 0}$, defined in analogy to $\mathscr{C}$ above, satisfies $(0)$ as well and, according to the criterion (7), we have a linear homeomorphism $(E, \varrho(\mathscr{C})) \cong\left(E^{\prime}, \varrho\left(\mathscr{C}^{\prime}\right)\right)$. Next we show:

(8) If there is a linear bijection $f: E \rightarrow E^{\prime}$ with $f\left(V_{i}\right)=V_{i}^{\prime}(i \geqq 0)$ then it is of the form $f=\left.l^{*}\right|_{E}$, where $l^{*} \in \mathrm{GL}\left(H^{*}\right)$ is the adjoint of a $l \in \mathrm{GL}(H)$ and the matrix of $l$ relative to $\left(h_{i}\right)_{i \geqq 1}$ is triangular.

Indeed, let $v_{i} \in V_{i-1} \backslash V_{i}(i \geqq 1)$. Construct a dual basis $\left(w_{i}\right)_{i \geqq 1}$ of $H$ by induction, $\left\langle v_{i}, w_{j}\right\rangle=\delta_{i j}(i, j \geqq 1)$. We have $w_{n}=\alpha_{1} h_{1}+\alpha_{2} h_{2}+\ldots+\alpha_{n} h_{n}$ with $0 \neq \alpha_{n} \in k$. If now $f\left(V_{i}\right)=V_{i}^{\prime} \quad(i \geqq 0)$ for some linear bijection $f: E \rightarrow E^{\prime}$ then $v_{i}^{\prime}:=f\left(v_{i}\right) \epsilon$ $V_{i-1}^{\prime} \backslash V_{i}^{\prime}$. Let $\left(w_{i}^{\prime}\right)_{i \geqq 1}$ be the dual basis of $\left(v_{i}^{\prime}\right)_{i \geqq 1}$ in $H$. Let $l \in \mathrm{GL}(H)$ be the map with $w_{i}^{\prime} \rightarrow w_{i}(i \geqq 1)$. One readily verifies that $f=\left.l^{*}\right|_{E}$ and that the matrix of $l$ has the desired diagonal shape.

By way of contradiction assume that there exists an $f$ enjoying (8). Let $p: H \rightarrow H_{-}$be the projection along $H_{+}, q: H^{*} \rightarrow H_{-}^{*}$ the projection along $H_{+}^{*}$, $t:=\left.p \circ l\right|_{H_{-}^{*}}$. Then the adjoint of $t$ is $t^{*}=\left.q \circ l^{*}\right|_{H_{-}^{*}}$. Now $t^{*}: H_{-}^{*} \rightarrow H_{-}^{*}$ is bijective, for the matrix of $t$ (relative to $\left(h_{2 i-1}\right)_{i \geqq 1}$ ) is obtained from the matrix of $l$ by cancelling rows and columns of even index and, therefore, is a triangular matrix with nonzero diagonal coefficients. Ergo $t$ and $t^{*}$ are invertible. We now obtain a contradiction as follows. Pick $y \in H_{-}^{*} \backslash M$. There is $z \in H_{-}^{*}$ with $t^{*}(z)=y$ by the invertibility of $t^{*}$; thus $z \in E$. Furthermore $y=q \circ l^{*}(z)$ so that $l^{*}(z)=y+x$ for some $x \in H_{+}^{*}$. Therefore $f(z)=l^{*}(z) \ddagger E^{\prime}$, a contradiction.

Remark 2. By abuse let us say that $X \subset(E, \varrho(\mathscr{C}))$ is closed if and only if the image of $X$ under the projection $E \rightarrow E / \cap \mathscr{C}$ is closed in the associated (hausdorff) quotient topology $\varrho$ of $\varrho(\mathscr{C})$. We see that $X$ is closed if and only if the filters $\mathscr{U}(x, X)$ in (5) are principal for all $x \in E$. Unfortunately, there are no "nice" cases $(E, \varrho(\mathscr{C}))$ where all subspaces $X$ would be closed; e.g. $S:=\oplus_{i \geqq 1} S_{i}$, where $V_{i} \oplus S_{i}=V_{i-1}(i \geqq 1)$, is always dense in $(E, \varrho(\mathscr{C}))$. Now, in the case of countable $\operatorname{dim} E$ one never runs into trouble with $\varrho(\mathscr{C})$ because such spaces $E$ can be exhausted by finite dimensional $X$ which are, of course, always closed. (In lieu of our distributivity (3), $\left(3^{\prime}\right)$ above it was possible in [9, p. 114] to postulate a stronger distributivity which required in particular $\varrho$-closedness for the domains of all partial mappings considered [18].) 


\section{The mapping problem in orthogonal geometry}

II. 1. On the problem. We now assume the vector space $E$ to be equipped with a nondegenerate (orthosymmetric) sesquilinear form $\Phi: E \times E \rightarrow k$. Let $\mathcal{O}=\mathcal{O}(E, \Phi) \subset \mathrm{GL}(E)$ be the subgroup of elements with respect $\Phi$ (orthogonal group or group of isometries $E \rightarrow E$ ). We are now looking for a $T \in \mathcal{O}$ which induces the given lattice isomorphism $\eta: \mathscr{V} \rightarrow \mathscr{V}^{\prime}$ of I.1.

In order to make evident the novelty of geometric situations encountered in dimensions $>\aleph_{0}$ we repeat a fact mentioned in [8]: Whereas in each dimension $\leqq \aleph_{0}$ there is precisely one isometry class of nondegenerate symmetric bilinear forms over $\mathbf{C}$ there are $2^{\aleph_{1}} \geqq \aleph_{2}$ isometry classes of such forms in dimension $\aleph_{1}$. Similar results hold for nondegenerate alternating forms which, in dimensions $\leqq \aleph_{0}$, are considered to be without gestalt.

In particular, the abundance of isometry classes of alternate forms in dimensions $>\aleph_{0}$ testifies to the extent of geometric complications that have nothing to do with the arithmetic of the base field.

Everything that follows could be phrased for symmetric forms over any field $k$ which has the property of allowing for only one isometry class of nondegenerate forms in dimension $\aleph_{0}$. In [9, Appendix 1 to Chapter II] many classes of such $k$ are listed. However, in order to stress the new geometric features of the uncountable we stay mostly with the simplest case, to wit, alternate forms. A few definitions will be stated for arbitrary forms. (In [5] and [17] the lattice method is applied to anisotopic forms; in [5] the lattices are infinite. [10] and [15] treat of bilinear forms in characteristic 2 . Dimensions are invariably $\aleph_{0}$.)

II. 2. The prediagonal forms. Let $(E, \Phi)$ be a sesquilinear space and $\gamma$ an ordinal. We call $(E, \Phi) \gamma$-diagonal if there exists an orthogonal decomposition $E=\oplus_{\imath}^{\perp} E_{\iota}$ with $\operatorname{dim} E_{\imath}<\aleph_{\gamma}$ for all $l ;$ a sesquilinear space which is isometric to a subspace of a $\gamma$-diagonal space is called $\gamma$-prediagonal; 1-diagonal spaces are also 0-diagonal and, trivially, the other way round; hence for $\gamma \in\{0,1\}$ we simply speak of diagonal and prediagonal forms or spaces. A diagonal space is always an orthogonal sum of lines and planes [9, p. 63]. The basic theory about $\gamma$-diagonal and prediagonal forms can be found in [4].

The classification of prediagonal spaces is solved in [8] for dimensions $<\boldsymbol{\aleph}_{\omega_{0}}$. Theorems 8,10,11 below will treat of certain cases of larger dimensions.

II. 3. The diagonal hull. Let $(E, \Phi)$ be a sesquilinear space, nondegenerate and of dimension $\aleph_{\alpha}(\alpha>0), \alpha$ an ordinal. The linear topology $\sigma_{\gamma}(\Phi)$ on $E$ is defined by the 0 -neighbourhood filter basis

$$
\left\{X \perp \mid X \in \mathscr{L}(E) \text { and } \operatorname{dim} X<\aleph_{\gamma}\right\} \quad(\gamma \geqq 0),
$$

whereas the linear topology $\tau_{\gamma}(\Phi)$ on $E$ is defined by the basis

$$
\left\{X \perp \mid X \in \mathscr{L}(E) \text { and } \operatorname{dim} X \leqq \aleph_{\gamma}\right\} \quad(\gamma \geqq 0) .
$$


We have $\tau_{\gamma}(\Phi)=\sigma_{\gamma+1}(\Phi)$; for the closure $\sigma_{\gamma} X$ of a subspace $X \subset\left(E, \sigma_{\gamma}(\Phi)\right)$ we have, in the case of a limit number,

$$
\lim (\lambda) \Rightarrow \sigma_{\lambda} X=\bigcap_{\gamma<\lambda} \sigma_{\gamma} X=\bigcap_{\gamma<\lambda} \tau_{\gamma} X .
$$

If $\Phi$ is nondegenerate then $\sigma_{\gamma}(\Phi), \tau_{\gamma}(\Phi)$ are hausdorff; $\sigma_{\gamma}(\Phi)$ (or $\tau_{\gamma}(\Phi)$ ) is discrete when $\gamma>\alpha(\gamma \geqq \alpha$, respectively). Each $(\gamma+1)$-diagonal space is complete in the topology $\tau_{\gamma}(\Phi)$. Conversely, we have the beautiful Theorem [16, p. 245]: Let $\gamma \geqq 0$ be a fixed ordinal and $(E, \Phi)$ a nondegenerate $(\gamma+1)$-prediagonal space. Then there exists, uniquely determined up to isometry, a smallest $(\gamma+1)$-diagonal overspace $(\widetilde{E}, \widetilde{\Phi}) ; \widetilde{E}$ is the completion of the space $\left(E, \tau_{\gamma}(\Phi)\right)$; the completion topology $\tilde{\tau}_{\gamma}(\Phi)$ coincides with $\tau_{\gamma}(\widetilde{\Phi})$ and $\operatorname{dim} \widetilde{E}=\operatorname{dim} E$.

II. 4. Further conditions on the lattices. Each $T \in \mathcal{O}(E, \Phi)$ operates on $\mathscr{L}(E)$ and commutes with the operations $\perp$ (taking the orthogonal) and $\tau_{\gamma}$ (forming the $\tau_{\gamma}(\Phi)$-closure) for all $\gamma \geqq 0$. It is therefore appropriate to consider in the mapping problem II. 1 but lattices $\mathscr{V}$ that are stable under these operations (cf. [11]). For $V \in \mathscr{L}(E)$ we define $\mathscr{V}(V)$ to be the complete sublattice of $\mathscr{L}(E)$, stably generated by $(0), V, E$ under the operations $+, \cap, \perp, \tau_{\gamma}(\gamma \geqq 0)$.

Examples. Let $E$ be diagonal, $E^{1}=(0), \operatorname{dim} E=\aleph_{\alpha}$. In the cases $\alpha=0,1,2$ the lattice $\mathscr{V}=\mathscr{V}(V)$ is distributive and finite, $|\mathscr{V}| \leqq 14$ if $\alpha=0[12, \mathrm{p}$. 11], $|\mathscr{V}| \leqq 34$ if $\alpha=1$ ([1], [2]), $|\mathscr{V}| \leqq 88$ if $\alpha=2$ ([8]); the bounds indicated are reached for suitable $V$. The lattices $\mathscr{V}(V)$ are unknown for $\alpha \geqq 3 .^{*}$ ) (See Problems 1,2 in II. 7.) However, $\mathscr{V}(V)$ is known for arbitrary $\alpha$ when $V$ is $\tau_{0}(\Phi)$-dense in $E$, it is the $\alpha+2$ chain

$$
E=\tilde{V}=\sigma_{1} V \supseteqq \sigma_{2} V \supseteqq \ldots \supseteqq \sigma_{i} V \supseteqq \ldots \supseteqq \sigma_{\omega_{0}} V \supseteqq \sigma_{\omega_{0}+1} V \supseteqq \ldots \supseteqq \sigma_{\alpha} V \supseteqq V \supseteqq(0) .
$$

II. 5. How can one classify prediagonal forms? The uniqueness of the diagonal hull easily yields the following fundamental fact: If $V$ is isometric to a prediagonal $V^{\prime}$ then there exists an isometry $T$ from $E=\tilde{V}$ onto $E^{\prime}=\tilde{V}^{\prime}$ which maps the chain $\mathscr{V}(V)$ onto $\mathscr{V}\left(V^{\prime}\right)$, i.e. $T$ induces a lattice isomorphism $\eta: \mathscr{V}(V) \rightarrow \mathscr{V}\left(V^{\prime}\right)$ which, obviously, preserves rank and commutes with $\perp$ and all closure operators $\tau_{\gamma}, \sigma_{\gamma}$. Thus we see that the classification of $\boldsymbol{\aleph}_{\alpha}$-dimensional prediagonal forms is equivalent to solving our mapping problem II. 1 for $\mathscr{V}, \mathscr{V}^{\prime}$ descending chains of the kind (10). (Cf. Remark 11.) These chains are infinite when $\alpha \geqq \omega_{0}$. As already mentioned in the Introduction we shall discuss only the typical case $\alpha=\omega_{0}$. The case $\alpha<\omega_{0}$ is solved in [8]. We begin by showing that the chain (10) can be entirely arbitrary:

II. 6. The chain (10) of closures of a prediagonal $V$ has no special properties.

*) Note added in proof. When $\alpha=3$ the lattice is known and $\left|\mathscr{V}^{\circ}\right| \leqq 957$ ([20]). If $\alpha \geqq 4$ we know that $|\mathscr{V}|=\infty([19])$; the lattices are not known. 
For the sake of clarity in the description of our construction we restrict ouiselves to proving the following theorem only. It can easily be generalized.

Theorem 3. Let $(E, \Phi)$ be nondegenerate and of dimenion $\aleph_{\omega_{0}}$, $\Phi$ symmetric and admitting an orthogonal basis. Assume that we are given an orthogonal decomposition $E=W \oplus H, \operatorname{dim} W=\aleph_{1}$, and an $\omega_{0}$-chain in $W, W=W_{0} \supset W_{1} \supset W_{2} \supset \ldots$ with $\operatorname{dim} W_{n} / W_{n+1}=1, \bigcap_{n<\omega_{0}} W_{n}=(0)$. In $E$ there exists a subspace $V$ such that its $\tau_{n}(\Phi)$-closures are

$$
\tau_{n} V=V \oplus W_{n} \quad\left(n \leqq \omega_{0}\right) .
$$

Remark 3. From Lemma 2 and Theorem 3 one gathers that the cardinals obtained from (10) by taking dimensions of quotients are a long way from pinning down the isometry type of $V$ when $\alpha \geqq \omega_{0}$.

II. 7. Proof of Theorem 3. It will be divided into several paragraphs.

7.1. In each $W_{n}$ we choose a basis $\left\{w_{n, \gamma} \mid 0 \leqq \gamma<\omega_{1}\right\}$ such that $w_{n, \gamma} \in$ $W_{n+\gamma} \backslash W_{n+\gamma+1}$ for all $\gamma<\omega_{0}$.

7.2. $H$ is spanned by an orthogonal basis [4, p. 1578 Theorem 4]. We introduce orthogonal decompositions: $H=\oplus^{\perp}\left\{H_{n} \mid n<\omega_{0}\right\}, \operatorname{dim} H_{n}=\aleph_{n+1}, H_{n}=\oplus^{\perp}\left\{H_{n, \gamma} \mid \gamma<\omega_{1}\right\}$ $\operatorname{dim} H_{n, \gamma}=\aleph_{n+1}$. In each $H_{n, \gamma}$ we choose an orthogonal basis $\left\{e_{n, \gamma}(\lambda) \mid 0 \leqq \lambda<\omega_{n+1}\right\}$.

7.3. Let $V$ be the span of all

$$
w_{n, \gamma}+e_{n, \gamma}(\lambda), \quad 0 \leqq \lambda<\omega_{n+1}, \quad 0 \leqq \gamma<\omega_{1}, \quad 0 \leqq n<\omega_{0} .
$$

7.4. We set $D_{n, \gamma}:=\operatorname{span}\left\{e_{n, \gamma}(\lambda)-e_{n, \gamma}(\mu) \mid \lambda, \mu<\omega_{n+1}\right\}, \quad D_{n}:=\oplus^{\mathcal{L}}\left\{D_{n, \gamma} \mid \gamma<\omega_{1}\right\}$. Hence $D_{n} \subset H_{n} \cap V$.

7.5. We define spaces $C_{n, m} \subset H_{n}$ for all $n, m$ with $0 \leqq n \leqq m<\omega_{0}$. To each linear combination $\sum_{\gamma} c_{\gamma} w_{n, \gamma}$ which falls into the space $W_{m}$ we assign - with the same scalars $c_{\gamma}$ - the element $\sum_{\gamma} c_{\gamma} e_{n, \gamma}(0) \in H_{n} ; C_{n, m}$ is defined as the span of these assigned elements. It is evident that

$$
\begin{gathered}
C_{n, m} \supseteqq C_{n, m+1} \supseteqq \ldots ; \operatorname{dim} C_{n, m} / C_{n, m+1}=1, \\
\cap C_{n, m}=(0), \quad C_{n, m} \cap D_{n}=(0) .
\end{gathered}
$$

7.6. We assert that the $\tau_{n}(\Phi)$-closures of $V$ are given by

$$
\begin{gathered}
\tau_{0} V=W_{0} \oplus H=E, \\
\tau_{n} V=W_{n} \oplus\left[C_{0, n} \oplus D_{0}\right] \oplus\left[C_{1, n} \oplus D_{1}\right] \oplus \ldots \oplus\left[C_{n-1, n} \oplus D_{n-1}\right] \\
\oplus H_{n} \oplus H_{n+1} \oplus H_{n+2} \oplus \ldots \\
\oplus \operatorname{span}\left\{w_{0}^{\prime}{ }_{0}+e_{0,0}(0), w_{0,1}+e_{0,1}(0), \ldots, w_{0, n-1}+e_{0, n-1}(0),\right. \\
w_{1,0}+e_{1,0}(0), \ldots, w_{1, n-2}+e_{1, n-2}(0), \\
\vdots \\
\left.w_{n-1,0}+e_{n-1,0}(0)\right\}
\end{gathered}
$$


7.7. In order to justify the direct sum between "span $\{\ldots\}$ " and the rest in the expression for $\tau_{n} V$ in 7.6 one makes systematic use of the normalization made in 7.1 for those $w_{n, \gamma}$ with $\gamma<\omega_{0}$.

7.8. On the inclusion $\supseteqq$ in 7.6: Because $\operatorname{dim} H_{n, \gamma}>\aleph_{n}$, each $w_{n, \gamma} \in W_{n}$ is a $\tau_{n}(\Phi)$-clusterpoint of $V, W_{n} \subset \tau_{n} V$. Thus $H_{n} \subset \tau_{n} V$. Likewise, $H_{m} \subset \tau_{n} V$ for all $m>n$. Nontrivial are only the inclusions $C_{r, n} \subset \tau_{n} V$ for $r<n$. Let

$$
x=\sum_{\gamma} c_{\gamma} e_{r, \gamma}(0) \in C_{r, n}, \quad \text { so } \quad \sum c_{\gamma} w_{r, \gamma} \in W_{n}
$$

by 7.5 . Ergo

$$
x-\sum c_{\gamma}\left(w_{r, \gamma}+e_{r, \gamma}(0)\right)=-\sum_{\gamma} c_{\gamma} w_{r, \gamma} \in W_{n}, \quad x \in W_{n}+V \subset \tau_{n} V .
$$

7.9. We show next that

$$
W \cap V=(0), \quad W \cap \tau_{n} V=W_{n} \quad(n \geqq 1) .
$$

The first assertion follows directly from the definition in 7.3. Let then $n \geqq 1$ and $y \in W \cap \tau_{n} V$. If $X=W \oplus H_{0} \oplus \ldots \oplus H_{n-1}$ then $U:=X^{\perp}=H_{n} \oplus H_{n+1} \oplus \ldots$ is a $\tau_{n}(\Phi)$ neighbourhood, $(y+U) \cap V \neq \emptyset$. Pick $u \in U$ with $y+u \in V$. By 7.3 there is $w \in W_{n}$ such that $w+u \in V$. Thus we obtain $y-w \in W \cap V=(0)$ so $y=w \in W_{n}$.

7.10. On the inclusion $\leqq$ in 7.6: Let $x \in \tau_{n} V$. By 7.8 we may modify $x$ and assume $x=w+\sum_{j=0}^{n-1} \sum_{\gamma} c_{j, \gamma} e_{j, \gamma}(0)$ for certain scalars $c_{j, \gamma}$. Fix a pair $(j, \gamma)$. By the choice of the basis $\left\{w_{j, 0}, \ldots, w_{j, \gamma}, \ldots\right\}$ in 7.1 there exist, for $\gamma \geqq n-j$, scalars $d_{j, s}(0 \leqq s \leqq n-j-1)$ such that

$$
w_{j, \gamma}-\sum_{s=0}^{n-j-1} d_{j, s} w_{j, s} \in W_{n},
$$

so

$$
e_{j, \gamma}(0)-\sum_{s=0}^{n-j-1} d_{j, s} e_{j, s}(0) \in C_{j, n} \subseteq \tau_{n} V
$$

We may thus assume without loss of generality that $c_{j, \gamma}=0$ if $\gamma \geqq n-j$. In the space $\operatorname{span}\left\{w_{0,0}+e_{0,0}(0), \ldots, w_{n-1,0}+e_{n-1,0}(0)\right\} \subset V$ (compare 7.6) there exists therefore a vector $y$ such that $x-y \in W$. Since $x-y \in \tau_{n} V$ also, we have $x-y \in W \cap \tau_{n} V=W_{n}$. This establishes the inclusion $\subseteq$ in 7.6 and the proof of 7.6 is complete.

7.11. From 7.6 we obtain by counting that

$$
\operatorname{dim} \tau_{n} V / \tau_{n+1} V=1 \quad\left(n<\omega_{0}\right) .
$$

7.12. Since $V \oplus W_{n} \subseteq \tau_{n} V$ and $V \oplus W_{0}=V \oplus W=E=\tau_{0} V$ and because $\operatorname{dim}\left(V \oplus W_{n}\right) /\left(V \oplus W_{n+1}\right)=1=\operatorname{dim} \tau_{n} V / \tau_{n+1} V$ by 7.11 , we obtain inductively that $V \oplus W_{n}=\tau_{n} V$. This terminates the proof of Theorem 3 .

Remark 4. Let $E$ be an orthogonal sum of $\aleph_{\omega_{0}}$ nondegenerate finite dimensional spaces $E_{\imath} \neq(0), \mathscr{B}_{\imath}$ a basis in $E_{l}, \mathscr{B}:=\cup_{\imath} \mathscr{B}_{l}$. The hyperplane $V:=$ span $\left.b-b^{\prime} \mid b, b^{\prime} \in \mathscr{B}\right\}$ has $\tau_{n} V=E$ for all $n<\omega_{0}$. By taking external orthogonal 
sums of $d$ copies of this example one obtains examples of prediagonal $V$ with

$$
\tilde{V}=\sigma_{1} V=\sigma_{\omega_{0}} V \supset V \supset(0), \operatorname{dim} \sigma_{\omega_{0}} V / V=d .
$$

By using this example and Theorem 3 to form orthogonal sums one can obtain many new examples (Cf. III. 5).

Problem 1. Show that $\mathscr{V}(V)$ is infinite when $\alpha \geqq 4$ (see II. 4 for the definition of $\mathscr{V}(V))$. A possible line of attack would be to use the technique of the proof of Theorem 3 to "produce" four previously fixed "independent" elements inside $\mathscr{V}(V)$; they would generate an infinite sublattice under + and $\cap$ alone. See also [13], [14].**)

Problem 2. $\mathscr{V}(V)$ when $\alpha<3$.**)

II. 8. The orbit of a chain under the group $\mathrm{GL}(E)$ is larger than the orbit under $\mathcal{O}(E, \Phi)$. Let $(E, \Phi)$ be an orthogonal sum of $2^{\aleph_{0}}$ hyperbolic planes and let the base field $k$ have $|k| \leqq 2^{\aleph_{0}}$. We are going to construct reduced chains $\mathscr{C}=\left(V_{i}\right)_{i<\omega_{0}}, \mathscr{C}^{\prime}=\left(V_{i}^{\prime}\right)_{i<\omega_{0}}$ in $\mathscr{L}(E)$ with property $(0)$ and such that

(j) for each ordinal $\gamma \geqq 0$ with $\leqslant \gamma<2 \aleph_{0}$ the spaces $V_{i}, V_{i}^{\prime}$ are $\tau_{\gamma}(\Phi)$-dense in $E\left(i<\omega_{0}\right)$

(jj) $(E, \varrho(\mathscr{C}))$ and $\left(E, \varrho\left(\mathscr{C}^{\prime}\right)\right)$ are complete.

The natural lattice isomorphism $\eta: \mathscr{C} \rightarrow \mathscr{C}^{\prime}$ preserves rank, obviously, and commutes with all operations $\tau_{\gamma}, \sigma_{\gamma}$ (cf. II. 3). Furthermore, by (jj) it is clear that $\eta$ is induced by an element $T \in \mathrm{GL}(E)$. We assert

Theorem 4. There are chains $\mathscr{C}, \mathscr{C}^{\prime}$ with $(0),(\mathrm{j})$, (jj) such that $\eta$ is induced by an element $T \in \mathrm{GL}(E)$ but not by any element of $\mathcal{O}(E, \Phi)$.

Proof. For a fixed chain $\mathscr{C}$ with $(0)$ and (jj) we construct two forms $\Phi, \Psi$ on $E$ such that (j) holds for either form and such that both $(E, \Phi)$ and $(E, \Psi)$ are orthogonal sums of hyperbolic planes. Further, no $T \in \mathrm{GL}(E)$ with $T V_{i}=$ $V_{i}\left(i<\omega_{0}\right)$ will be an isometry $(E, \Phi) \rightarrow(E, \Psi)$. If $\varphi:(E, \Phi) \rightarrow(E, \Psi)$ is any fixed isometry and $\mathscr{C}^{\prime}:=\varphi \mathscr{C}=\left(\varphi V_{i}\right)_{i<\omega_{0}}$ then one obtains the assertion of the theorem.

The number of mappings $T \in \mathrm{GL}(E)$ with $T V_{i}=V_{i}\left(i<\omega_{0}\right)$ is precisely $\aleph_{\alpha}:=|k|^{\aleph_{0}}=2 \aleph^{\aleph_{0}}$; for these maps are describable by triangular $\aleph_{0} \times \aleph_{0}$-matrices (cf. the proof in I. 6). Let $\mathscr{E}=\left\{T_{\xi} \mid 0 \leqq \xi<\omega_{\alpha}\right\}$ be an enumeration of these $T$.

We define recursively vectors $x_{\xi}, \bar{x}_{\xi} \in E, 0 \leqq \xi<\omega_{\alpha}$. To this end we subdivide the $\omega_{0}$-sections of $\omega_{\alpha}$ into adjacent intervals of four elements each; we demand

(i) $\left(x_{\xi}\right)_{\xi},\left(\bar{x}_{\xi}\right)_{\xi}$ are linearly independent families,

(ii) for all limit numbers $\lambda<\omega_{\alpha}$ we have $x_{\lambda+4 n+r} \in V_{n} \backslash V_{n+1}$ (for all $n<\omega_{0} ; r=0,1,2,3$ ),

**) Note added in proof. Problem 1 has been solved along the line indicated. For $\alpha>3$ the lattices are infinite, in general, but their structure is not known $([19,20])$. 
(ii) for all limit numbers $\lambda<\omega_{\alpha}$ we have $\bar{x}_{\lambda+4 n+r} \in V_{n} \backslash V_{n+1}$ (for all $n<\omega_{0} ; r=0,1,2,3$ ),

(iii) for all limit numbers $\lambda<\omega_{\alpha}$ and all $n<\omega_{0}$ we have

$$
T_{\lambda+n}: x_{\lambda+4 n+r} \rightarrow \bar{x}_{\lambda+4 n+r} \quad(r=0,1,2,3) .
$$

Assume that $x_{\xi}, \bar{x}_{\xi}$ for $\xi<\lambda+4 n$ are already constructed, and let $F:=$ $\operatorname{span}\left\{x_{\xi}, T_{\lambda+n}^{-1}\left(\bar{x}_{\xi}\right) \mid \xi<\lambda+4 n\right\}, \quad \operatorname{dim} F<\operatorname{dim} E$. Because $\operatorname{dim} \operatorname{span}\left(V_{n} \backslash V_{n+1}\right)=\aleph_{\alpha}$ linearly independent elements $y_{0}, y_{1}, y_{2}, y_{3} \in V_{n} \backslash V_{n+1}$ can be found such that $F \cap \operatorname{span}\left\{y_{0}, y_{1}, y_{2}, y_{3}\right\}=(0) . \quad$ Set $\quad x_{\lambda+4 n+r}:=y_{r}, \quad \bar{x}_{\lambda+4 n+r}:=T_{\lambda+n}\left(x_{\lambda+4 n+r}\right), \quad(r=$ $0,1,2,3)$. By construction (i), (ii), (iii) are satisfied; (ii) is satisfied because $V_{n}, V_{n+1}$ are invariant under $T_{\lambda+n}$. On $X:=\operatorname{span}\left(x_{\xi}\right)_{\xi<\omega_{x}}$ and $\bar{X}:=\operatorname{span}\left(\bar{x}_{\xi}\right)_{\xi<\omega_{x}}$ we define forms $\Phi, \Psi$ according to the table

$$
\begin{array}{l|l}
\Phi\left(x_{\lambda+4 n}, \quad x_{\lambda+4 n+1}\right)=1 & \Psi\left(\bar{x}_{\lambda+4 n}, \bar{x}_{\lambda+4 n+2}\right)=1 \\
\Phi\left(x_{\lambda+4 n+2}, x_{\lambda+4 n+3}\right)=1 & \Psi\left(\bar{x}_{\lambda+4 n+1}, \bar{x}_{\lambda+4 n+3}\right)=1 \\
\text { all other products zero } & \text { all other products zero }
\end{array}
$$

One may always carry out the construction of the $x_{\xi}$ in such a way that $\operatorname{dim} E / X$, $\operatorname{dim} E / \bar{X}$ are of equal infinite dimension; hence $\Phi$ and $\Psi$ may each be extended to a nondegenerate symplectic form on all of $E$ by letting $X, \bar{X}$ be orthogonal summands.

It is evident that no $T \in \mathrm{GL}(E)$ with $T V_{i}=V_{i}\left(i<\omega_{0}\right)$ can be an isometry $(E, \Phi) \rightarrow(E, \Psi)$.

In order to verify (j) let be given $\gamma<\alpha, V_{n}, z \in E$ and a $\tau_{\gamma}(\Phi)$-neighbourhood $z+U^{\perp}\left(\operatorname{dim} U \leqq \aleph_{\gamma}\right)$. There is a limit number $\lambda<\omega_{x}$ such that $x_{\xi} \in U^{\perp}$ for all $\xi \geqq \lambda$. By (ii) there is a linear combination

$$
u=\alpha_{0} x_{\lambda}+\alpha_{1} x_{\lambda+4}+\ldots+\alpha_{n-1} x_{\lambda+4(n-1)}
$$

such that $z-u \in V_{n}$; so $\left(z+U^{\perp}\right) \cap V_{n} \neq \emptyset$ as was to be shown. The argument for $\tau_{\gamma}(\Psi)$ is mutatis mutandis the same.

II. 9. The orbits under GL $(E)$ are larger than the orbits under the group $\mathscr{H}(E, \Phi)$ of all linear $\tau_{0}(\Phi)$-homeomorphisms $E \rightarrow E$. The technique used in II.8. can be further developed: we can show

Theorem 5. Let $k, E, \Phi$ be as in II.8. There are chains $\mathscr{C}, \mathscr{C}^{\prime}$ with (0), (j), (jj) such that $\eta$ is induced by an element $T \in \mathrm{GL}(E)$ but not by any element of $\mathscr{H}(E, \Phi)$.

Proof. As in the foregoing proof we fix a given chain with properties (0) and (ji) and construct two symplectic forms $\Phi$ and $\Psi$ on $E$.

Let $\omega_{\alpha}=\bigcup_{\boldsymbol{\imath}<\omega_{\alpha}} P_{\boldsymbol{\imath}}$ be a fixed partitioning of $\omega_{\alpha}$ such that $\left|P_{\imath}\right|=\aleph_{\alpha}$ and each $P_{\imath}$ is a union of some $\omega_{0}$-sections of $\omega_{\alpha}$. Let $\mathscr{E}$ be as in II.8.

We then construct $\left(x_{\xi}\right)_{\xi},\left(\bar{x}_{\xi}\right)_{\xi}$ with (i), (ii), (ii) just as before; instead of (iii) however we demand that 
(iii*) for all limit numbers $\lambda<\omega_{\alpha}$ and all $n<\omega_{0}$ we have

$$
T_{\xi}: x_{\lambda+4 n+r} \rightarrow \bar{x}_{\lambda+4 n+r} \quad(r=0,1,2,3)
$$

where $P_{\xi} \ni \lambda+4 n+r$. In order words, whereas in the previous proof each $T_{\xi} \in \mathscr{E}$ was used to map one quadruple of $x$ 's only, we will here use each $T_{\xi}$ to map $\aleph_{x}$ many quadruples.

The form $\Phi$ is defined on $E$ as before (II.8 (iv)).

The form $\Psi$ is defined as follows. Let $\mathscr{B}_{\xi}^{\prime}:=\left\{\bar{x}_{\lambda+4 n+r} \mid r=0,1 ; \lambda+4 n+r \in P_{\xi}\right\}$, $\mathscr{B}_{\xi}^{\prime \prime}:=\left\{\bar{x}_{\lambda+4 n+r} \mid r=2,3 ; \lambda+4 n+r \in P_{\xi}\right\}$. Define $\Psi_{\xi}^{\prime}$ on span $\mathscr{B}_{\xi}^{\prime}$ to be the transported form: $\Psi_{\xi}^{\prime}(\bar{x}, \bar{y}):=\Phi\left(T_{\xi}^{-1} \bar{x}, T_{\xi}^{-1} \bar{y}\right)$ for all $\xi<\omega_{\alpha}$. (This part is to guarantee that (j) will hold for $\Psi$ as well.) In order to define $\Psi_{\xi}^{\prime \prime}$ on span $\mathscr{B}_{\xi}^{\prime \prime}$, transform the $\aleph_{\alpha}$ elements of $\mathscr{B}_{\xi}^{\prime \prime}$ by some invertible matrix that is row-finite but has nondenumerably many non-zero entries in some of its columns. Let $\overline{\overline{\mathscr{B}}}_{\xi}=\left\{\overline{\bar{x}} \mid \bar{x} \in \cap \mathscr{B}_{\xi}^{\prime \prime}\right\}$ be the resulting basis of $\operatorname{span} \mathscr{B}_{\xi}^{\prime \prime}$. The $\overline{\bar{x}}_{\mathrm{s}}$ naturally come in pairs (as transforms of pairs $\bar{x}_{\lambda+4 n+2}, \bar{x}_{\lambda+4 n+3}$ ). We declare these pairs of $\overline{\bar{x}}$ 's to form a symplectic basis of $\operatorname{span} \mathscr{B}_{\xi}^{\prime \prime}$ thereby defining $\Psi_{\xi}^{\prime \prime}$. The upshot is the following: If $\Phi$ is transported under $T_{\xi}$ onto span $\mathscr{B}_{\xi}^{\prime \prime}$ then this form will make the basis $\overline{\overline{\mathscr{B}}}_{\xi}$ a noncontinuous $\tau_{0}$-basis $[3$, p. 30 Satz 11].

Because $\left(\bar{x}_{\xi}\right)_{\xi<\omega_{\alpha}}=\bigcup_{\xi<\omega_{\alpha}} \mathscr{B}_{\xi}^{\prime} \cup \mathscr{B}_{\xi}^{\prime \prime}$ is a disjoint union we can form the external orthogonal sum of all $\Psi_{\xi}^{\prime}, \Psi_{\xi}^{\prime \prime}$ and obtain the required symplectic form $\Psi$ on all $E$ as in the proof of Theorem 4 .

By the very construction it is clear that no element $T \in \mathscr{E}$ is a homeomorphism $\left(E, \tau_{0}(\Phi) \stackrel{\sim}{\longrightarrow}\left(E, \tau_{0}(\Psi)\right)\right.$.

Problem 3. Study the orbits under $\mathscr{O}(E, \Phi), \mathscr{H}(E, \Phi)$ of the chain $\mathscr{C}$ given in (10) in the special situation: $\alpha=\omega_{0}$ and $\operatorname{dim} \sigma_{n} V / \sigma_{n+1} V=1 \quad\left(n<\omega_{0}\right)$ and $(E, \varrho(\mathscr{C}))$ is complete. (The last assumption implies that all such chains $\mathscr{C}$ form just one single orbit under GL $(E)$.)

Theorems 4 and 5 nicely demonstrate that the classification problems in the metric situation are not merely shadows of projective or affine problems; they certainly have their own status. The mapping problems I. 1 and II. 1 are of equal standing. The solution of one does not solve the other.

\section{Solution of the mapping problem in special metric cases}

III.1. On the lattice method in the presence of descending chains. The recursive method developed in [8] in order to solve the mapping problem II. 1. for finite lattices is not, in general, adequate if the lattice contains infinite descending chains. This fact clearly manifests itself already in the simplest case when $\mathscr{V}$ is a chain $\mathscr{C}=\left(V_{i}\right)_{i<\omega_{0}}$, reduced or not, with property $(0)$ and $\operatorname{dim} E=\aleph_{1}$. For, the partial 
maps $\varphi$ whose limit ought to induce a lattice isomorphism

$$
\eta: \mathscr{C} \rightarrow \mathscr{C}^{\prime}
$$

are homeomorphisms with respect to $\varrho(\mathscr{C}), \varrho\left(\mathscr{C}^{\prime}\right)$ by (1). Further, whatever course the recursion may take, after countably many steps supplements of $V_{i+1}$ in $V_{i}$ for all $i$ will, by necessity, be contained in $\operatorname{dom} \varphi$; i.e. $\operatorname{dom} \varphi$ will be $\varrho(\mathscr{C})$-dense in $E$. Hence, the limit map, if such there is, will be uniquely determined modulo $\cap \mathscr{C}$ by this partial map $\varphi$.

Therefore, in order that the recursion will not be hopelessly stuck after the first few steps when $\operatorname{dim} E / \cap \mathscr{C}>\aleph_{0}$, additional assumptions on the chains are mandatory.

In [8] the class of finite (distributive) lattices was treated. By the same arguments the class of (distributive) countable lattices with DCC can be taken care of.

In the following we shall solve the mapping problem for two more classes of lattices. As these allow for infinite descending chains of a certain kind, the range of applicability of the results is greatly increased. filter if

III.2. Almost principal filters. We say that a filter $\mathscr{G} \subset \mathscr{V}$ is almost a principal

$$
\min \{\operatorname{dim} G / \cap, \mid G \in \mathscr{G}\} \leqq \aleph_{0}
$$

In this section we treat of complete lattices $\mathscr{V} \subset \mathscr{L}(E)$ all of whose filters $\mathscr{G}$ satisfy condition (11) and the well-known condition (4). We assert

Theorem 6. Let $(E, \Phi)$ be a diagonal alternate nondegenerate space of dimension $\aleph_{\alpha}, 0<\alpha<\omega_{1}, \mathscr{V}$ a countable, complete, distributive sublattice of $\mathscr{L}(E)$ satisfying (4) and stable under the operations $\perp$ and $\tau_{\gamma}(\gamma<\alpha)$. Let $\mathscr{V}^{\prime}$ be another lattice of the same kind in $\mathscr{L}(E)$ and

$$
\eta: \mathscr{V} \rightarrow \mathscr{V}^{\prime}
$$

a lattice isomorphism preserving rank and commuting with $\perp$ and $\tau_{\gamma}(\gamma<\alpha)$. In order that $\eta$ be induced by an isometry $T \in \mathcal{O}(E, \Phi)$ it is sufficient that all filters $\mathscr{G} \subset \mathscr{V}$ satisfy condition (11). Thus, in the class of complete distributive lattices with (4) and (11) described here, the orbits under the orthogonal group are characterized by cardinal invariants (namely the family of indices $\operatorname{dim} A / B, A \supset B$ in $\mathscr{V}$ ).

The proof of Theorem 6 follows essentially the line of the proof of [8, Theorem 2]. We refrain here from describing the details. We should rather like to stress some points of major interest.

III.3. Remarks about Theorem 6. 5. The reasons for the condition " $\alpha<\omega_{1}$ " are described in [8, Remarks 1,9]. The discussion of this other great barrier $\aleph_{\omega_{1}}$ will not be taken up here. 
Remark 6. (The role of condition (11).) One tries to follow the setup in I. 4 and construct the required isometry $T$ as a limit of partial maps $\varphi: X \rightarrow X^{\prime}$ of a suitable subfamily $\mathscr{F}_{2}(\eta) \subset \mathscr{F}_{1}(\eta)$. Let $x \in E \backslash X$. The fact that the filter

$$
\mathscr{M}(x, X):=\{A \in \mathscr{V} \mid x \in X+A\}
$$

be principal is crucial for the possibility of extending $\varphi$ to $X \oplus(x)$. That this will indeed be the case is taken care of as follows. In countably many steps we first construct a $\left(\varphi_{*}: X_{*} \rightarrow X_{*}^{\prime}\right) \in \mathscr{F}_{1}(\eta)$ with the following properties:

(a) $\operatorname{dim} X_{*} \leqq \aleph_{0}$, (b) if $\mathscr{G}$ is a filter in $\mathscr{V}$ and $D:=\cap \mathscr{G}$ then $X_{*}+D \supseteqq G$ for some $G \in \mathscr{G}$. It is then clear that for any extension $\left(\varphi: X \rightarrow X^{\prime}\right) \in \mathscr{F}_{1}$ of $\varphi_{*}$ the filter $\mathscr{M}(x, X)$ is principal. Set $\mathscr{F}_{2}(\eta):=\left\{\varphi \in \mathscr{F}_{1}(\eta) \mid \varphi \supseteqq \varphi_{*}\right\}$. We are left to show how $\varphi_{*}$ is found.

For each filter $\mathscr{G} \subset \mathscr{V}$ let $D:=\cap \mathscr{G}$; by (11) there is $S \in \mathscr{L}(E)$ with $\operatorname{dim} S \leqq \aleph_{0}$ such that $G:=D+S \in \mathscr{G}$. As $|\mathscr{V}| \leqq \aleph_{0}$ the number of all possible pairs $(D, G) \in \mathscr{V} \times \mathscr{V}$ is countable. If, for each $\mathscr{G} \subseteq \mathscr{V}$, we pick such an $S$, then their sum $S_{*}$ is of countable dimension. We require $S_{*} \subset \operatorname{dom} \varphi_{*} ; \varphi_{*}$ can be obtained as the limit of a chain $\left(\varphi_{i}: X_{i} \rightarrow X_{i}^{\prime}\right)_{i<\omega_{0}}$ in $\mathscr{F}_{1}(\eta)$ with $\operatorname{dim} X_{i}<\infty$ throughout. For finite $\operatorname{dim} X_{i}$ the filter $\mathscr{M}\left(x, X_{i}\right)$ is invariably principal.

III.4. Compatible pairs $(\mathscr{V}, \Phi)$. Let $(E, \Phi)$ be a sesquilinear space and $\mathscr{V} \subset \mathscr{L}(E)$ a sublattice. $\mathscr{V}$ and $\Phi$ are said to be compatible, if $(E, \Phi)$ admits at least one orthogonal decomposition $E=\oplus_{I}^{\perp} E_{l}, \operatorname{dim} E_{l} \leqq \aleph_{0}$, such that the following condition is satisfied:

(12) If $E^{+}=\oplus_{J} E_{l}$ with $J \subset I$ and $|J|<|I|$ and $\mathscr{G} \subset \mathscr{V}$ is a filter then the $\varrho(\mathscr{G})$ closure of $E^{+}$is $E^{+}+\cap \mathscr{G}$ (i.e. the image of $E^{+}$in $E / \cap \mathscr{G}$ is closed in the (hausdorff) quotient topology $\varrho$ associated with $\varrho(\mathscr{G})$ ).

Remark 7. It is not true that, for a compatible pair $(\mathscr{V}, \Phi)$, condition (12) holds for every orthogonal decomposition $E=\oplus E_{\imath}\left(\operatorname{dim} E_{\imath} \leqq \aleph_{0}\right)$ of $E$; for this would mean that each orthogonal summand $F$ of $E\left(E=F+F^{\perp}\right)$ would be $\varrho$-closed, and to this one can find counterexamples.

If $(V, \Phi)$ is a nondegenerate prediagonal space of a dimension $\aleph_{n}, n<\omega_{0}$, then the lattice (chain) $\mathscr{V}(V)$ in $\mathscr{L}(\tilde{V})$ is finite, hence $(\mathscr{V}(V), \widetilde{\Phi})$ is a compatible pair. Less obvious examples will be considered in Sections III.5, III.6.

Theorem 7. Let $(E, \Phi), \mathscr{V}, \mathscr{V}^{\prime}$ be as in Theorem 6 and let $\eta: \mathscr{V} \rightarrow \mathscr{V}^{\prime}$ be a lattice isomorphism that commutes with $\perp$ and $\tau_{\gamma}(\gamma<\alpha)$ and that preserves $\operatorname{dim} X / X_{0}$ for each compact join-irreducible $X \neq(0)$ in $\mathscr{V}$ and its (unique) antecedent $X_{0}$. In order that $\eta$ be induced by an isometry $T \in \mathcal{O}(E, \Phi)$ it is sufficient that $\mathscr{V}, \mathscr{V}^{\prime}$ are compatible with $\Phi$.

The proof of Theorem 7 follows the general scheme detailed in [8]. Difficulties caused by the presence of infinite descending chains are offset by compatibility 
which forces the crucial filters $\mathscr{M}(x, X)$ to be principal. Preservation of rank is needed only in Section 2.5 of [8] and only for pairs $X, X_{0}$ as indicated in Theorem 7.

Remark 8. Theorem 7 is an instance where preservation of rank follows from equality among certain selected indices by virtue of compatibility. Does this mean that, in the compatible case, well ordered descending chains $\mathscr{V} \subset \mathscr{L}(E)$ necessarily have additivity of supplements in the sense of the following definition?

Definition 1. The well ordered descending chain $\mathscr{V}=\left(X_{\imath}\right)_{0 \Xi_{\imath}<\gamma}$ in $\mathscr{L}(E)$ enjoys additivity of supplements if and only if there exists a family $\left(S_{\imath}\right)_{0 \unlhd_{1}<\gamma}$ with

$$
X_{\imath+1} \oplus S_{\iota}=X_{\imath} \quad(0 \leqq \imath<\gamma)
$$

and such that for arbitrary $v<\mu<\gamma$ we have

$$
X_{\mu} \oplus \underset{v \leqq i<\mu}{\bigoplus} S_{\imath}=X_{v} .
$$

Remark 9. Given a family $\left(S_{\imath}\right)_{\imath}$ with (13) it is sometimes believed that the fact whether or not the equality (14) holds is a property of $\mathscr{V}$. This is of course not so: in dimension $\aleph_{0}$ each chain $\left(X_{\imath}\right)_{0 \leqq_{l}<\omega_{0}+1}$ admits some families $\left(S_{1}\right)_{l}$ with (13) that make (14) true and others with (13) that falsify (14).

Problem 5. Answer the question enunciated in Remark 8 (for the particular chains $\mathscr{V}=\mathscr{V}(V)$ considered in Theorem 8 below the answer is affirmative).

Problem 6. Classify compatible pairs $(\mathscr{V}, \Phi)$.

III.5. Examples of compatible pairs (orthogonal decompositions). We describe here a particularly simple brand of prediagonal spaces $V$ that have the property that their lattices (chains) $\mathscr{V}(V)(10)$ are compatible with the form on the diagonal hull $\tilde{V}=E$. As we shall be able to prescribe indices Theorem 7 will be applicable: the examples offer themselves as normal forms (see the following section).

Let $d_{\imath}(1 \leqq l \leqq \alpha)$ be arbitrary cardinals with $0 \leqq d_{\imath} \leqq \aleph_{\alpha}$; let furthermore $U_{\gamma}$ be any prediagonal space of dimension $\boldsymbol{\aleph}_{\gamma}(\gamma>0)$ with

$$
\operatorname{dim} \tilde{U}_{\gamma} / U_{\gamma}=1, \quad \tau_{v} U_{\gamma}=\tilde{U}_{\gamma}, \quad \text { for all } 0 \leqq v<\gamma,
$$

(there are such spaces by Remark 4). We form external orthogonal sums

$$
V:=\underset{1 \leqq \imath \leqq \alpha}{\oplus \perp} d_{l} \cdot U_{l} .
$$

We then read off:

$$
\begin{gathered}
\tau_{v} V=\sigma_{v+1} V=\bigoplus_{1 \leqq l \leqq v} d_{\imath} \cdot U_{\imath} \oplus \underset{v<\imath \leqq \alpha}{\bigoplus} d_{l} \cdot \tilde{U}_{l} \quad(0 \leqq v<\alpha), \\
\bigcap_{v<\lambda} \tau_{v} V=\sigma_{\lambda} V=\bigoplus_{1 \leqq \imath<\lambda} d_{\imath} \cdot U_{\imath} \oplus \bigoplus_{\lambda \leqq l \leqq \alpha} d_{\imath} \cdot \tilde{U} \quad(\lim (\lambda) \quad \lambda \leqq \alpha), \\
\operatorname{dim} \sigma_{v} V / \sigma_{v+1} V=d_{v} \quad(2 \leqq v+1 \leqq \alpha), \\
\operatorname{dim} \sigma_{\mu} V / \sigma_{v} V=\sum_{\mu \leqq l<v} d_{\imath} \quad(1 \leqq \mu<v \leqq \alpha), \\
\operatorname{dim} \sigma_{\alpha} V / V=d_{\alpha} .
\end{gathered}
$$


It would be very easy, but cumbersome, to write the last three formulae in terms of the $\tau$ 's. We finish by establishing compatibility: As for $E=\oplus_{\imath<\omega_{\alpha}}^{\perp} E_{\imath}$ we choose a decomposition which is obtained by throwing together similar decompositions of the $\tau_{0}(\Phi)$-completions of the single summands of $V$ in (16), $\operatorname{dim} E_{\imath} \leqq \aleph_{0}$. We consider a subspace $E^{+}=\oplus F_{\imath}$ with $F_{\imath} \subset E_{\imath}$ and a limit number $\lambda$ with $0<\lambda \leqq \alpha$. We shall show that

$$
E^{+}+\sigma_{\lambda} V \supseteqq \bigcap_{v<\lambda}\left(E^{+}+\sigma_{v} V\right) .
$$

Since the converse inclusion is trivial this tells that $E^{+}$is "closed" under $\varrho\left(\left(\sigma_{v} V\right)_{1 \leqq \nu \leqq \lambda}\right)$. Let $p$ be an element of the intersection. There is $l_{0}<\lambda$ such that $p=p_{1}+p_{2}, p_{1} \in L:=\bigoplus_{l} \geqq_{0} E_{l}, p_{2} \in \oplus_{l} \geqq \lambda E_{l}$. We have $p_{2} \in \sigma_{\lambda} V$ by (18), hence $p_{1}$ is also an element of the intersection ( $\varrho$-closure of $E^{+}$). Let $\pi: E \rightarrow L$ be the projection along $L^{\perp}$. As $F_{\imath} \subset E_{\imath}$ we have $\pi E^{+} \subset E^{+}, \pi \tau_{v} V \subset \tau_{v} V$. For each $v<\lambda$ there is a decomposition $p_{1}=x(v)+y(v)$ with $x(v) \in E^{+}, y(v) \in \sigma_{v} V$. Ergo $p_{1}=\pi p_{1}=$ $\pi x(v)+\pi y(v) \in\left(E^{+} \cap L\right)+\left(\sigma_{v} V \cap L\right)$. We fix a $v, l_{0}<v<\lambda$, and find by (17) $\sigma_{v} V \cap L=$ $\oplus_{l} \geqq_{0} d_{i} \cdot U_{i}$; thus $p_{1} \in E^{+}+V$. Altogether $p=p_{1}+p_{2} \in E^{+}+\sigma_{\lambda} V$.

III.6. Normal forms of prediagonal spaces. Let $\mathrm{E}\left(\operatorname{dim} \mathrm{E}=\aleph_{\alpha}\right)$ be any diagonal space; it can be written as an orthogonal sum of lines and planes

$$
E_{\imath}=\oplus \perp F_{\xi}, \quad 1 \leqq \operatorname{dim} F_{\xi} \leqq 2 .
$$

Choose any basis $\mathscr{B}_{\xi}$ of $F_{\xi}$ and set $\mathscr{B}:=\cup \mathscr{B}_{\xi}$. If $\mathscr{L}>0$ then the hyperplane

$$
U_{l}:=\operatorname{span}\left\{b-b^{\prime} \mid b, b^{\prime} \in \mathscr{B}\right\}
$$

is prediagonal and not diagonal [7, p. 104, Satz 1 and its obvious generalization to the case of hyperbolic planes]; it is the simplest specimen of its kind. Since it enjoys property (15) it can be used in (16). We obtain:

Theorem 8. Let $(V, \Phi)$ be a nondegenerate prediagonal alternate space of dimension $\aleph_{\alpha}\left(0<\alpha<\omega_{1}\right)$. The statements (i) and (ii) are equivalent:

(i) The chain $\mathscr{V}(V) \subset \mathscr{L}(\tilde{V})(c f .(10))$ is compatible with the form $\tilde{\Phi}$ on $\tilde{V}$.

(ii) $V$ has the form (16), with $U_{\imath}$ as in (23), $\tilde{V}=\oplus_{l} \Xi_{\alpha} d_{l} \cdot E_{l}$, with uniquely determined cardinals $d_{\imath}(1 \leqq \imath \leqq \alpha)$ given by (19) and (21).

Proof. "(ii) $\Rightarrow($ i)" is the content of III.5. Conversely, given the chain $\mathscr{V}(V)$ we can make the $d_{\imath}$ in (16) equal to the indices of corresponding neighbouring elements in $\mathscr{V}(V)$. An application of Theorem 7 yields "(i) $\Rightarrow$ (ii)".

Corollary. Let $(V, \Phi)$ be as in (i) of Theorem 8. Then we have additivity of supplements in the sense of Definition 1 (Remark 8); in particular we have "additivity of indices" in the sense of (20).

Remark 10. By using Theorem 3 we can easily give examples of prediagonal $V$ such that $\mathscr{V}(V)$ fails to have additivity. In these cases $\mathscr{V}(V)$ cannot be compatible with the form on $\tilde{V}$. By Theorem 8 , these $V$ cannot be orthogonally de- 
composed into the building blocks $U_{\imath}$. Of course, $\alpha \geqq \omega_{0}$ in these cases. In contrast, if $\alpha<\omega_{0}$ the requirement of compatibility is empty and we have

Theorem 9. Every nondegenerate prediagonal alternate space $V$ of dimension $\aleph_{n}, n<\omega_{0}$, is either diagonal or of the shape

$$
V=d_{1} \cdot U_{1} \stackrel{\perp}{\oplus} \ldots \stackrel{\perp}{\oplus} d_{n} \cdot U_{n} \quad\left(\operatorname{dim} U_{i}=\aleph_{i}\right),
$$

and its diagonal hull $E=\tilde{V}$ is

$$
E=d_{1} \cdot E_{1} \stackrel{\perp}{\oplus} \ldots \stackrel{\perp}{\oplus} d_{n} \cdot E_{n},
$$

where $E_{i} \supset U_{i}$ is given by (22), (23). Furthermore,

$$
d_{i}=\operatorname{dim} \tau_{i-1} V / \tau_{i} V \quad(1 \leqq i<n), \quad d_{n}=\operatorname{dim} \tau_{n-1} V / V ;
$$

the $n$ cardinals $0 \leqq d_{i} \leqq \aleph_{n}$, together with $\operatorname{dim} V=\aleph_{n}$, form a complete set of independent invariants for the isometry class of $V$.

If $(\mathscr{V}(V), \tilde{\Phi})$ is compatible for $V$, a nondegenerate alternate prediagonal space of dimension $\aleph_{\alpha}\left(\alpha<\omega_{1}\right)$, then we learn from Theorem $8,(17),(18)$ and from the explicit shape of the $U_{l}$ in (22), (23) that with (10) there is associated an ascending chain $\left(S_{\imath}\right)_{1 \geqq_{l}<\alpha+1}$ of totally isotropic "supplements" with

$$
\begin{aligned}
& \sigma_{\imath} V_{\imath} \oplus S_{\imath}=\tilde{V}, \quad V \oplus S_{\alpha+1}=\tilde{V}, \quad S_{\imath} \text { is } \sigma_{0}(\tilde{\Phi}) \text { closed in } \tilde{V} \\
& \text { (and hence } \sigma_{1}(\tilde{\Phi}) \text {-closed and thus } \sigma_{1}(\tilde{\Phi}) \text {-complete), } 1 \leqq \imath \leqq \alpha+1 .
\end{aligned}
$$

Problem 6. Does each nondegenerate alternate prediagonal space admit a totally isotropic supplement in its diagonal hull?

A solution of Problem 6 is important for practical calculations; e.g., there is the following lemma which we mention without proof:

Lemma 3. Let $W$ be any nondegenerate prediagonal space and $\bar{W}$ its $\mu$-diagonal hull, $\mu \geqq 0$ a fixed ordinal. Let $U \subset W$ be a $\mu$-diagonal nondegenerate subspace which is maximal in the sense that $\operatorname{dim} W / U$ is minimal. If $W$ admits a totally isotropic supplements in $\bar{W}$ then $\operatorname{dim} \bar{W} / W=\operatorname{dim} W / U$.

If $\bar{W}$ is anisotropic in Lemma 3 we may very well have $\operatorname{dim} \bar{W} / W<\operatorname{dim} W / U$; see [7, p. 110, Satz 5].

III. 7. Topological characterization of the isometry classes of certain prediagonal spaces. From an isometry $(E, \Phi) \cong\left(E^{\prime}, \Phi^{\prime}\right)$ between two arbitrary sesquilinear spaces we obtain of course a homeomorphism $\left(E, \tau_{0}(\Phi)\right) \cong\left(E^{\prime}, \tau_{0}\left(\Phi^{\prime}\right)\right)$. When do we have the converse?

$$
\left(E, \tau_{0}(\Phi)\right) \cong\left(E^{\prime}, \tau_{0}\left(\Phi^{\prime}\right)\right) . \Rightarrow .(E, \Phi) \cong\left(E^{\prime}, \Phi^{\prime}\right) .
$$

That there are interesting instances of (25) is demonstrated by Theorem 8 and

Theorem 10. If $(V, \Phi)$ and $\left(V^{\prime}, \Phi^{\prime}\right)$ are alternate spaces of the shape described in (16) then the implication (25) is valid. In particular, for the nondegenerate, alternate, 
prediagonal spaces of dimension $\aleph_{n}$ with $n<\omega_{0}$ isometry classes and $\tau_{0}$-homeomorphy classes coincide.

Proof. If $\left(V, \tau_{\mu}(\Phi)\right) \cong\left(V^{\prime}, \tau_{\mu}\left(\Phi^{\prime}\right)\right)$ holds for a particular value of $\mu$, then it holds for all larger values as well. Thus, if it holds for $\mu=0$ we obtain equality of all indices, $\operatorname{dim} \sigma_{\mu} V / \sigma_{\mu+1} V=\operatorname{dim} \sigma_{\mu} V^{\prime} / \sigma_{\mu+1} V^{\prime}$. Hence the assertion.

In [3, p. 37, IV. 2] an example of a homeomorphism $\left(E, \tau_{0}(\Phi)\right) \cong\left(E^{\prime}, \tau_{0}\left(\Phi^{\prime}\right)\right)$ is given with one space $(E, \Phi)$ prediagonal and not the other. A newer result by Bäni [6] tells that, in contrast to $\tau_{0}$, the topology $\sigma_{0}$ "decides" on prediagonality: if under a homeomorphism $\left(E, \sigma_{0}(\Phi)\right) \cong\left(E^{\prime}, \sigma_{0}\left(\Phi^{\prime}\right)\right)$ one space is prediagonal then so is the other, whence

Theorem 11. Let $(V, \Phi)$ be a nondegenerate alternate prediagonal space such that the chain $\mathscr{V}(V)$ is compatible with the form $\tilde{\Phi}$ on the diagonal hull $\tilde{V}$. Let $\left(V^{\prime}, \Phi^{\prime}\right)$ be any alternate space (over the same base field) such that $\left(V, \sigma_{0}(\Phi)\right) \cong$ $\left(V^{\prime}, \sigma_{0}\left(\Phi^{\prime}\right)\right)$. If $\operatorname{dim} V<\aleph_{\omega_{1}}$ then there is an isometry $(V, \Phi) \cong\left(V^{\prime}, \Phi^{\prime}\right)$.

If we do not assume compatibility (which means passing to dimensions $\geqq \aleph_{\omega_{0}}$ ) then there are results which point to quite different directions. We give just one illustration:

(26) There are prediagonal spaces $(E, \Phi),\left(E^{\prime}, \Phi^{\prime}\right)$ of dimension $\aleph_{\omega_{0}}$ such that all closures $\tau_{l} E, \tau_{l} E^{\prime}\left(0 \leqq l<\omega_{0}\right)$ are isometric yet $(E, \Phi)$ and $\left(E^{\prime}, \Phi^{\prime}\right)$ are not isometric.

Problem 7. What classes of spaces (other than that described in Theorem 10) satisfy the implication (25)?

Remark 11. Theorem 10 is particularly satisfying in that there is no reference to the diagonal hull of the spaces to be classified. We are aware of the fact that behind our treatment of prediagonal spaces $V$ (II.5) there lurks the possibility to treat these spaces from within, i.e. without plunging them into their hulls. One should first try to compute the invariants $\operatorname{dim} \sigma_{\imath} V / \sigma_{\imath+1} V$ inside $V$ by expressing them as generalized defects in the spirit of [7, Chapter III].

\section{References}

[1] Амport, P.: Teilraumverbände in überabzählbardimensionalen Sesquilinearräumen. - Ph. D. Thesis University of Zurich, 1978, 1-64.

[2] Амport, E.: Modelle für Teilraumverbände in überabzählbardimensionalen Sesquilinearräumen. - Ph. D. Thesis University of Zurich, 1981, 1-117.

[3] BÄNI, W.: Sesquilineare Formen und lineare Topologien. - Ph. D. Thesis University of Zurich, $1975,1-43$.

[4] BÄNI, W.: Linear topologies and sesquilinear forms. - Comm. Algebra 5 no. 14, 1977, 1561 1587. 
[5] BäNI, W.: Applications of the lattice method to infinite dimensional hermitian spaces (subspaces in hermitian spaces of countable dimension). - Habilitationsarbeit 84, Universität Zürich, 1981, 1-119. Part I (pp. 1-59) is to appear in Math. Z.

[6] BÄNI, W.: Extending sesquilinear forms. - Preprint 1976, $1-4$.

[7] Gross, H.: Der Euklidische Defekt bei quadratischen Räumen. - Math. Ann. 180, 1969, 95-137.

[8] Gross, H.: The lattice method in the theory of quadratic spaces of non-denumerable dimensions. - J. Algebra 75, 1982, 23-42.

[9] Gross, H.: Quadratic forms in infinite dimensional vector spaces. - Progress in Mathematics, 1. Birkhäuser, Boston-Basel-Stuttgart, 1979.

[10] Gross, H., and H. A. Keller: On the non trace-valued forms. - Adv. in Math., 42, 1981, $179-195$.

[11] HaApasalo, L.: Von Vektorraumisometrien induzierte Verbandsisomorphismen zwischen nicht orthostabilen und nicht distributiven Vektorraumverbänden. - Ann. Acad. Sci. Fenn. Ser. A I Math. Dissertationes 37, 1981, 1-86.

[12] Kaplansky, I.: Forms in infinite-dimensional spaces. - An. Acad. Brasil. Ciênc. 22, 1950, 1-17.

[13] LOMECKY, Z.: Computer generated lattices. - University of Zurich, 1980,

[14] LoMeCKy, Z.: Algorithms for compution of gree lattices. In: Computer algebra. EUROCAM 82, European computer, algebra conference. Marseille, France, April 1982. Lecture notes in computer science, vol. 144. Springer Verlag, Berlin, Hiedelberg, New York (1982) 223-230.

[15] MoResI, R.: Untersuchungen in abzählbar-dimensionalen nichtspurwertigen $\varepsilon$-hermitischen Räumen. - Ph. D. Thesis, University of Zurich, 1980, 1-50.

[16] OGG, E.: Die abzählbare Topologie und die Existenz von Orthogonalbasen in unendlichdimensionalen Räumen. - Math. Ann. 188, 1970, 233-250.

[17] SaARImÄKI, M.: Zur Klassifikation von Paaren dichter Teilräume in hermiteschen Räumen von abzählbarer Dimension. - Ann. Acad. Sci. Fenn. Ser. A I Math. Dissertationes 34, 1981, 1-58.

[18] Schwabe, G.: Der Reiter und der Bodense (1826) in "Proben der deutschen Poesie seit dem XVI. Jahrhundert" von Wilhelm Wackernagel, 3. Aufluge Basel, Schweighausersch Verlagsbuchandlung 1876.

[19] Hross, H., Lomeczky, Z., Schuppti, R: Lattice problems originating in quadratic space theory. Preprint. April 1983 pp. 1-34.

[20] SCHUPPLI, R.: Untersuchungen zu quadratischen Räumen kleiner überalzählbarer Dimension. PhD. Thesis Univ. of Zürich, 1983, 1-99.

\author{
University of Zürich \\ Department of Mathematics \\ Rämistrasse 74 \\ CH-8001 Zürich \\ Switzerland
}

Received 22 June 1982 